\title{
"See and Treat" in Pronto Soccorso: aspetti legali e responsabilità professionale infermieristica
}

\author{
"See and Treat" in the Emergency Department: \\ legal aspects and professional nursing responsibility
}

\author{
Cristiano Radice $^{1} \quad$ Monica Ghinaglia ${ }^{2} \quad$ Renzo Doneda $^{2} \quad$ Giovanna Bollini $^{3}$
}

\begin{abstract}
RIASSUNTO
Larticolo si propone di analizzare gli aspetti legali inerenti la responsabilità professionale infermieristica nella presa in carico autonoma del paziente con un problema di salute minore che accede ad un percorso di "See and Treat" all'interno del Pronto Soccorso, attraverso una revisione della letteratura e un'analisi della normativa italiana in tema di esercizio professionale. Recenti studi hanno dimostrato che la gestione dei pazienti che accedono al Pronto Soccorso con problemi di salute minori in un'area dedicata, gestita da infermieri specificamente formati, ha consentito di ridurre i tempi di attesa per la visita medica ed il tempo di permanenza nel Dipartimento di Emergenza. Diversi studi hanno dimostrato i benefici dell'utilizzo del Emergency Nurse Practitioner (ENP) in termini di riduzione del tempo di attesa per la visita medica, con un considerevole aumento del grado di soddisfazione del paziente, mantenendo adeguati standard di qualità nella risposta ai problemi clinici minori. Il "See and Treat" rappresenta una possibile strategia di risposta al sovraffollamento, insieme all'adozione di protocolli avanzati di post-triage, ponendosi come principale obiettivo la riduzione del tempo di attesa in Pronto Soccorso per i pazienti classificati come codici minori, evitando così un improprio utilizzo delle risorse materiali e professionali. L'infermiere di "See and Treat" si configura come un infermiere esperto, adeguatamente formato, che opera nel rispetto di protocolli clinico-assistenziali condivisi tra medici ed infermieri. La normativa in tema di esercizio professionale non è in contrasto con l'introduzione di questa figura, anzi offre elementi per un ulteriore sviluppo professionale.
\end{abstract}

Parole chiave: See and Treat, Emergency Nurse Practitioner, traumi minori, infermieristica in emergenza

\section{ABSTRACT}

The article aim to analyze the legal aspects of professional responsibility in the autonomous nursing care of a patient with a minor health problem treated in a "See and Treat" area of the Emergency Department through a literature review and an analyses of the Italian legislation about professional exercise. Recent studies have shown that the treatment of the emergency patients affected by minor health problems in separated areas of the A\&E by skilled nurses proved to be effective in reducing time to medical examination and the overall time spent in the Emergency Department. Several studies have shown the positive effects of the Emergency Nurse Practitioner (ENP) in terms of reduction of time to medical examination with an increase in patient satisfaction, maintaining an adequate level of quality in the care of patients with minor health problems. The introduction of a "See and Treat" area, together with the institution of advanced post-triage protocols, represents a possible answer to the overcrowding of the Emergency Department. The aim is the reduction of waiting times and proper allocation of both material and professional resources. The "See and Treat" nurse represents an expert nurse, with an adequate level of competence, who acts in respect to the clinical protocols shared between physicians and nurses. The Italian legislation is not in contrast with the introduction of the "See and Treat" nurse, on the contrary it offers opportunities for further professional development.

Keywords: See and Treat, Emergency Nurse Practitioner, Minor Injuries Units, Emergency Nursing

INTRODUZIONE

$\mathrm{I}^{\mathrm{l}}$ sovraffollamento del Pronto Soccorso rappresenta un problema crescente ed ormai ben conosciuto che coinvolge, senza sostanziali differenze, tutti i Paesi industrializzati. Secondo i dati del Ministero della Salute, 400 abitanti su 1000 ogni anno si rivolgono

1 Infermiere legale e forense, Pronto Soccorso, Ospedale Niguarda Cà Granda di Milano. Corrispondenza: cristiano.radice@yahoo.it

2 Coordinatore infermieristico, Pronto Soccorso, Ospedale Niguarda Cà Granda di Milano

3 Dottore Magistrale in Scienze Infermieristiche ed ostetriche, Direttore D.I.T.R.A., Ospedale Niguarda Cà Granda di Milano al Pronto Soccorso, 250 dei quali per problemi di salute minori (Ministero della Salute, 2005). Da un lato non è ancora chiaro se l'aumento dei pazienti a bassa priorità assistenziale peggiori l'outcome dei pazienti con un grado di priorità più alto, dall'altro è stato dimostrato un considerevole aumento dei tempi di attesa prima della visita medica dei pazienti a maggiore criticità (McCarthy et al., 2009). È stata inoltre dimostrata una correlazione tra il sovraffollamento del Pronto Soccorso ed il verificarsi di errori, seppure non gravi, nelle diverse fasi del processo di cura (Fordyce et al., 2003). Da ciò deriva la necessità 
di prevedere per i pazienti che accedono al Pronto Soccorso con problemi di salute minori un opportuno ed adeguato percorso di cura, gestito da infermieri adeguatamente formati, nel rispetto di protocolli clinico-assistenziali, con lo scopo di ridurre i tempi di attesa prima della visita medica e di consentire una adeguata presa in carico dei pazienti ai vari livelli di criticità ed intensità di cura.

Nel Regno Unito, secondo i dati del Department of Health, nel primo trimestre del 2011 il 96,6\% dei pazienti accettati in Pronto Soccorso hanno dovuto attendere fino a 4 ore prima della presa in carico. Dei 3,4 milioni di pazienti che si sono rivolti al Pronto Soccorso in ospedali con un Dipartimento di Emergenza ed Accettazione di alta specializzazione, solamente il $25,5 \%$ ha poi richiesto un ricovero (Department of Health, 2011). Nell'ottobre 2001 la Reforming Emergency Care Strategy ha avviato un lungo programma di modernizzazione del sistema di emergenza sanitaria inglese, con l'obiettivo di trattare un maggior numero di pazienti, riducendo considerevolmente le liste di attesta, sia attraverso interventi migliorativi strutturali sia attraverso una riorganizzazione dell'intero sistema. Il 20\% degli ospedali inglesi hanno adottato il "See and Treat" come un efficace sistema per trattare i pazienti che si presentano in Pronto Soccorso con problemi di salute o traumi minori (National Health Service, 2003).

Il paziente viene valutato e trattato dal primo professionista sanitario disponibile, sia esso un medico o un infermiere specializzato, definito quest'ultimo Emergency Nurse Practitioner (ENP), una figura infermieristica presente sia nella realtà statunitense che in quella anglosassone (Advanced Nurse Practitioner o Advanced Practice Nurse), che si configura come un infermiere con competenze cliniche avanzate, una formazione universitaria post-base, in grado di erogare un'assistenza sanitaria di alta qualità, diagnosticando e trattando numerosi problemi di salute, unitamente alla promozione della salute e alla prevenzione delle malattie.

Le sue competenze cliniche comprendono una completa raccolta anamnestica, l'esame fisico del paziente, la prescrizione e l'interpretazione di indagini diagnostiche, inclusa la prescrizione di alcuni farmaci. E' pertanto in grado di assicurare un'appropriata gestione delle problematiche sanitarie del paziente, affetto da malattie acute e croniche come ad esempio il diabete, l'ipertensione arteriosa, alcune infezioni e traumi (American Academy of Nurse Practitioner, 2010; Royal College of Nurses, 2007).

L'articolo si propone di descrivere il "See and Treat", analizzando le origini e le competenze dell'Emergency Nurse Practitioner, valutandone poi l'applicabilità nel contesto normativo italiano.

\section{METODI}

La ricerca bibliografica è stata preceduta dalla lettura di documenti, articoli, siti internet inerenti il modello "See and Treat" diffuso in Inghilterra, così da poter meglio identificare quali termini utilizzare per impostare la stringa di ricerca. Inoltre si è tenuta in considerazione una precedente revisione della letteratura condotta da Bambi et al. (2008).

La ricerca bibliografica è stata condotta su PubMed, inizialmente si è verificata la presenza nel dizionario $\mathrm{MeSH}$ dei termini ricavati dalla lettura dei documenti sopra citati, in particolare: "see and treat", "minor injuries unit", "fast track", "emergency nurse practitioner" ed "emergency department". Nessuno dei termini è risultato presente nel dizionario $\mathrm{MeSH}$. Pertanto, ai fini della ricerca, si è scelto di utilizzare i seguenti due termini presenti nel $\mathrm{MeSH}$ :

- Emergency Service, Hospital (dipartimento ospedaliero responsabile dell'ammissione e del trattamento d'urgenza del paziente affetto da problematiche sia di tipo medico che chirurgico);

- Emergency Nursing (specializzazione dell'assistenza infermieristica diretta alla cura dei pazienti ammessi al dipartimento di emergenza).

Una prima ricerca su PubMed, utilizzando i due termini appena citati con l'operatore booleano OR, ha permesso di selezionare 43.796 fonti. Successivamente sono stati applicati i seguenti limiti: pubblicazione entro gli ultimi 5 anni; lingua italiana o inglese; soggetti umani adulti (>19 anni); disponibilità dell'abstract. Si sono così selezionate 4.784 fonti.

Una seconda ricerca su PubMed, utilizzando come testo libero i termini "see and treat", "minor injuries unit", "fast track", "emergency nurse practitioner" con l'operatore booleano OR applicando i medesimi limiti, ha consentito di selezionare 279 fonti.

Combinando le due ricerche utilizzando l'operatore booleano AND si sono ottenute 19 fonti, di cui 2 non risultano rintracciabili nel Sistema Bibliotecario Biomedico Lombardo (SBBL) e 5 non risultano attinenti all'argomento oggetto di studio. La stringa di ricerca utilizzata in PubMed è rappresentata in Figura I. Per aumentare i risultati della ricerca si è fatto ricorso alla funzione "related articles", al fine di poter identificare ulteriori fonti bibliografiche pertinenti che non fossero incluse nei limiti della stringa di ricerca.

("Emergency Service, Hospital" [MeSH] OR “Emergency Nursing" [MeSH]) AND

("see and treat" OR "minor injuries unit" OR "fast track" OR "emergency nurse practitioner")

Figura 1. Search di ricerca sul database PubMed, a cui sono stati applicati limiti: pubblicazione entro 5 anni; lingua italiana o inglese; soggetti umani adulti (>19 anni); disponibilità dell'abstract. 


\section{RISULTATI}

Il Pronto Soccorso riveste un ruolo di primaria importanza, sia nell'erogazione di prestazioni sanitarie urgenti per i pazienti critici, sia perché rappresenta un punto di riferimento per i pazienti affetti da problemi di salute minori o condizioni cliniche con caratteristiche di cronicità che non trovano adeguata risposta da parte dei servizi ambulatoriali e territoriali. L'efficacia e l'efficienza del servizio erogato dipendono dalla corretta classificazione del paziente al triage e vengono valutate in base al rispetto degli standard clinico-assistenziali previsti da diverse società scientifiche, come ad esempio il Canadian Triage and Acuity Scale National Working Group (Bullard et al., 2008; Warren et al., 2008). Numerosi autori sono concordi nell'affermare che l'afflusso di pazienti affetti da problemi di salute minori determini il sovraffollamento del Pronto Soccorso, con un considerevole aumento dei tempi di attesa per la visita medica, un aumento del carico di lavoro assistenziale e conseguentemente l'insoddisfazione da parte dell'utente (Quattrini et al., 2011). Per fronteggiare questo problema sono state sviluppate diverse strategie, tra cui l'introduzione di un ambulatorio di "See and Treat" dedicato al trattamento dei pazienti con problemi di salute minori, basandosi sul presupposto che un professionista sanitario esperto (medico o infermiere) possa essere in grado di valutare, trattare e dimettere in tempi brevi il paziente con un problema di salute minore (Rogers et al., 2004).

I prolungati tempi di attesa in Pronto Soccorso, oltre a determinare il sovraffollamento del Dipartimento di Emergenza, sono stati identificati come possibili potenziali cause di episodi di violenza verso i pazienti e gli operatori sanitari. Le lunghe attese rappresentano inoltre un costo per la società, poiché comportano una riduzione del tempo dedicato al lavoro, all'istruzione, alla famiglia (Kwa et al., 2008).

La gestione del paziente in "See and Treat" viene affidata ad un infermiere esperto, denominato Emergency Nurse Practitioner (ENP). Nella realtà italiana l'ENP potrebbe corrisponde ad un infermiere con competenze avanzate, in possesso di un master clinico di specializzazione o della laurea magistrale ${ }^{1}$, che abbia seguito uno specifico percorso di formazione e che, per i problemi clinici minori, operi nel rispetto di protocolli clinico-assistenziali di trattamento. Diversi studi in letteratura hanno dimostrato i benefici dell'utilizzo del ENP in termini di riduzione del tempo di attesa per la visita, con un considerevole aumento del grado di soddisfazione del paziente, mantenendo comunque adeguati standard di qualità seppur con un lieve aumento dei costi, legato al fatto che l'ENP visita 1-2 pazienti ogni ora, mentre lo standard previsto per il medico è di 3-4 pazienti ogni ora (Carter et al., 2007). Ciò sembra essere dovuto alla rigidità dei protocolli di triage che consentono di assegnare un limitato numero di pazienti all'area "See and Treat", oltre alla maggiore attenzione posta dal ENP all'informazione e all'educazione sanitaria. L'introduzione della figura dell'ENP può rivelarsi vantaggiosa, anche dal punto di vista economico, negli ospedali caratterizzati da un alto flusso di pazienti con complessità assistenziale medio-alta, poiché consente di utilizzare le risorse mediche solamente per la cura dei casi clinicamente più complessi (Carter et al., 2007; Ieraci et al., 2008).

Hart e Mirabella (2009) hanno condotto uno studio osservazionale, intervistando 300 pazienti che si sono rivolti a 3 distinti Dipartimenti di Emergenza, evidenziando che il $65 \%$ dei pazienti intervistati preferiva essere trattato da un ENP e che il $93 \%$ dei pazienti, che erano già stati trattati in precedenza da un ENP, erano soddisfatti del trattamento ricevuto. Risultati positivi sono stati ottenuti anche da Jennings et al. (2009), sottoponendo a 202 pazienti che si sono rivolti ad un trauma center di primo livello un questionario semivalidato a 16 domande espresse con una scala di Likert a 4 punti. Infine Nash et al. (2007) hanno condotto uno studio osservazionale retrospettivo, utilizzando un questionario costituito da 6 domande aperte con una scala di Likert a 4 punti, riportando un grado di soddisfazione pari a 3,71 per la qualità della visita ricevuta, 3,85 per la cortesia del personale sanitario, 3,45 per la durata del tempo di permanenza, 3,83 per la qualità dell'assistenza erogata, 3,87 per il rispetto della privacy e 3,78 come giudizio globale. Un ulteriore dato significativo è rappresentato da un basso tasso di ripresentazione nel Dipartimento di Emergenza nei 3 giorni successivi, pari al $3 \%$.

Sebbene non esista una specifica certificazione, l'Emergency Nurses Association ha sviluppato uno strumento di validazione delle competenze che dovrebbe possedere un ENP (Emergency Nurses Association, 2008). Rimane inoltre da sottolineare che anche l'American College of Emergency Physicians ha riconosciuto in un apposito documento l'importanza dell'utilizzo del ENP nell'ambito dell'assistenza in

1 - Legge n. 43/2006 "Disposizioni in materia di professioni sanitarie infermieristiche, ostetrica, riabilitative, tecnico-sanitarie e della prevenzione e delega al Governo per l'istituzione dei relativi ordini professionali”. Art. 6: Il personale laureato appartenente alle professioni sanitarie è articolato in: professionisti in possesso del diploma di laurea; professionisti coordinatori in possesso del master di primo livello in management o per le funzioni di coordinamento; professionisti specialisti in possesso del master di primo livello per le funzioni specialistiche; professionisti dirigenti in possesso della laurea specialistica. 
condizioni di emergenza (American College of Emergency Physicians, 2003).

Dalla revisione della letteratura condotta da Bambi et al. (2008) emergono le caratteristiche che dovrebbe possedere un ENP per garantire una efficace gestione dei pazienti con problemi di salute minori, in particolare: esperienza, buon senso, capacità di accettare le responsabilità, di riconoscere i propri limiti, e di chiedere aiuto.

\section{DISCUSSIONE}

La revisione della letteratura ha consentito di dimostrare il vantaggioso utilizzo dell'ENP nella gestione dei pazienti affetti da problematiche cliniche minori, evidenziato dalla riduzione del tempo di attesa per la visita medica e dall'alto livello di soddisfazione dei pazienti. Verranno ora presentate alcune considerazioni in merito all'applicabilità della figura dell'ENP in relazione al contesto normativo italiano.

In Italia la Regione Toscana ha elaborato nel 2007 un progetto sperimentale, identificando nel "See and Treat" una possibile risposta assistenziale alle urgenze minori (Regione Toscana, Delibera n. 958 del 17 dicembre 2007; Regione Toscana, Delibera n. 449 del 31 marzo 2010). L'obiettivo è ricondurre il Pronto Soccorso alla funzione propria, che è quella di garantire un trattamento sanitario nelle situazioni di emergenza-urgenza ed il ricovero appropriato, oltre alla diminuzione delle attese per i codici di bassa priorità, attraverso la creazione di un percorso di accesso facilitato, a gestione infermieristica, per i pazienti con problemi di salute minori. La finalità principale di tale sperimentazione è verificare la possibilità di un'ottimizzazione dei tempi di risposta all'utenza e delle risorse professionali a disposizione. I problemi trattabili in "See and Treat" sono stati individuati in base all'incidenza, alla gravità, agli eventuali interventi terapeutici necessari e alla co-presenza del medico-tutor e sono riportati in Tabella I.

Per quanto riguarda l'aspetto formativo, è previsto per l'infermiere assegnato all'area "See and Treat" il superamento di un corso della durata complessiva di 350 ore, considerando sia la formazione teorica che quella pratica, che consente l'acquisizione del titolo di Infermiere certificato in interventi di primo soccorso.

L'avvio della sperimentazione toscana ha suscitato diverse polemiche. Secondo l'Ordine dei Medici di Bologna "si assiste ad una tendenza di sviluppo di competenze e connessi comportamenti ed attività che dall'esecutività propria dell'infermieristico-assistenziale finiscono con l'assumere di fatto carattere infermieristico-medico, allorquando viene riconosciuta all'infermiere discrezionalità in campo diagnostico-terapeutico che non può di certo trovare legittimazione sotto una dichiarata copertura della finale supervisione medica", affermando inoltre che possa in realtà diventare un elemento favorente l'esercizio abusivo della professione medica, oltre che elemento di deresponsabilizzazione dell'infermiere (Ordine dei Medici Chirurghi e degli Odontoiatri di Bologna, 29 giugno 2010).

L'Ordine dei Medici di Bologna sostiene l'illegittimità del provvedimento adottato dalla Regione Toscana in violazione dell'Art. 117, comma 3, della Costituzione Italiana in tema di ripartizione delle competenze tra Stato e Regioni, in violazione della normativa sanitaria nazionale, dell'autonomia scientifica e deontologica della professione medica, ritenendo pertanto che possa configurarsi la fattispecie di reato previsto dall'Art. 348 Codice Penale riguardante l'esercizio abusivo della professione medica.

La Federazione dei Collegi IPASVI è intervenuta nel dibattito sostenendo che l'assistenza sanitaria non si struttura solamente in un insieme di processi diagnostici e terapeutici (peraltro resi concreti in cospicua

\begin{tabular}{|c|c|}
\hline Urgenze oftalmologiche & $\begin{array}{l}\text { Ecchimosi periorbitale senza disturbi della funzione visiva; congiuntivite; corpo estraneo congiuntiva- } \\
\text { le; emorragia sottocongiuntivale; irritazione da lenti a contatto }\end{array}$ \\
\hline Urgenze otorinolaringoiatriche & Tappo di cerume; otite esterna; corpo estraneo nell’orecchio; epistassi; corpo estraneo nel naso; rinite \\
\hline Urgenze odontostomatologiche & Lussazione ricorrente della mandibola; dolore dentario; problemi post-estrazione dentaria \\
\hline Urgenze gastroenterologiche & Singhiozzo isolato; diarrea acuta non ematica; reinserimento/disostruzione del sondino naso gastrico \\
\hline Urgenze urologiche & Infezione delle basse vie urinarie; sostituzione/ disostruzione del catetere vescicale \\
\hline Urgenze ginecologiche & Test gravidici di esclusione \\
\hline Urgenze muscolo-scheletriche & Torcicollo; lombalgia acuta ricorrente; dolore acuto monoarticolare \\
\hline Urgenze traumatologiche & $\begin{array}{l}\text { Contusioni minori degli arti; traumi delle dita delle mani e dei piedi non complicati; pronazione dolo- } \\
\text { rosa; ferite semplici e abrasioni; avulsione superficiale della punta del dito; rimozione di anello; rimo- } \\
\text { zione di amo da pesca; punture da animali marini e punture di insetti; intrappolamento nella lampo; } \\
\text { rimozione di punti di sutura }\end{array}$ \\
\hline Urgenze dermatologiche & $\begin{array}{l}\text { Dermatiti da contatto; ustioni solari; ustioni minori; geloni; orticaria; pediculosi; verruche; foruncoli; } \\
\text { cisti sebacea; idrosoadenite; infezioni del letto ungueale }\end{array}$ \\
\hline
\end{tabular}

Tabella I. Casistica di riferimento per la sperimentazione del "See and Treat" approvata dal gruppo di lavoro per il progetto di miglioramento del Pronto Soccorso. Osservatorio regionale sull'emergenza-urgenza della Regione Toscana. 
parte dall'infermiere), ma anche nella rilevazione e nella risposta appropriata ai molteplici bisogni che il paziente evidenzia o che l'infermiere autonomamente rileva nell'esercizio della sua quotidiana professionalità. Afferma inoltre che le riflessioni e l'approfondimento professionale, che hanno sostenuto gli importanti cambiamenti normativi già citati, si sono concretizzati nella definizione di metodi e strumenti per pianificare, gestire e valutare correttamente l'intervento assistenziale infermieristico, per rilevare e rispondere appropriatamente ai molteplici bisogni delle persone assistite nei diversi setting ospedalieri e territoriali (Silvestro, 2010).

La Società Italiana di Medicina d'EmergenzaUrgenza (SIMEU) ha preso le distanze da quanto sostenuto dall'Ordine dei Medici di Bologna, affermando che una tale posizione "mette in crisi l'attuale assetto organizzativo dei Dipartimenti di Emergenza-Urgenza, 118 e Pronto Soccorso [...]; mette in crisi la sicurezza del cittadino rispetto ad una sua tempestiva presa in carico da parte del personale infermieristico nelle situazioni di emergenza-urgenza; mette in crisi l'identità professionale degli infermieri che operano nei servizi di emergenza-urgenza non riconoscendo il ruolo fondamentale che da anni gli infermieri hanno assunto nel rispondere ai bisogni di salute dei cittadini; vanifica i tentativi di collaborazione interprofessionale medicoinfermiere oggi più che mai necessari nel settore dell'emergenza-urgenza, anche per il perdurante e progressivamente crescente problema del sovraffollamento" (SIMEU, 13 novembre 2010). Richiamando quanto disposto dal D.P.R. del 27 marzo 1992, dalla Legge n. 42/1999, dalla Legge n. 251/2000, la SIMEU attribuisce agli infermieri un ruolo primario nel soccorso territoriale e nel triage di Pronto Soccorso.

Le norme citate individuano, nel loro complesso, un ambito proprio, e pertanto esclusivo, di competenza infermieristica ed un ambito collaborativo con le altre professioni sanitarie.

Il già citato D.M. n. 739/1994 all'Art. 5 specifica che la formazione infermieristica post-base per la pratica specialistica deve essere intesa a fornire agli infermieri di assistenza generale delle conoscenze cliniche avanzate e delle capacità che permettano loro di fornire specifiche prestazioni infermieristiche in diverse aree: sanità pubblica, pediatrica, salute mentale, geriatrica, area critica.

È necessario, a questo punto, stabilire se le funzioni attribuite all'infermiere di "See and Treat" rientrino nell'ambito di competenza e quindi di autonomia della professione infermieristica, per verificare se sussistano o meno le condizioni di esercizio abusivo della professione medica. La Suprema Corte di Cassazione ha avuto modo di precisare che "solamente una fonte normativa può consentire a soggetti diversi da quelli esercitanti la professione di medico interventi invasivi sulla sfera corporale, sulla base di un ragionevole riconoscimento di competenze tecniche e professionali" (Corte di Cassazione, sez. VI, 21 febbraio 1997). La recente evoluzione normativa ha determinato un importante cambiamento, stabilendo le competenze di ciascuna figura professionale in base al possesso di specifiche conoscenze necessarie per il compimento di determinati atti. Pertanto il nuovo quadro normativo si presenta antitetico rispetto all'interpretazione fornita dalla Suprema Corte di Cassazione, la quale considerava vincolante per l'esercizio professionale il solo possesso del titolo di studio e l'abilitazione all'esercizio della professione, arrivando a considerare "irrilevanti la perizia, la capacità e l'abilità del soggetto", criteri che sono oggi ritenuti alla base dell'esercizio professionale (Benci, 1999). La dottrina giuridica e medico-legale ha analizzato il termine competenza riconoscendogli un significato non univoco, può infatti significare "compito, attività che si è tenuti a svolgere" oppure anche "capacità, abilità, conoscenza”. Nel primo caso un'attività sarebbe di competenza medica quando essa venga attribuita da una specifica norma, legge o regolamento al medico. Nel secondo caso un'attività sarebbe di competenza medica quando solamente le capacità, le abilità e le conoscenze che formano il curriculum professionale del medico siano in grado di porla in essere. A questo proposito occorre notare che non esiste alcun mansionario che elenchi con precisione le attività di esclusiva competenza medica, ad eccezione della formulazione di una diagnosi di patologia e della prescrizione terapeutica (Benci, 2008). L'Unione Europea dei Medici Specialisti (2007) ha adottato la seguente definizione di atto medico: "L'atto medico comprende tutte le azioni professionali, vale a dire le attività scientifiche, didattiche, formative ed educative, cliniche, medico tecniche compiute al fine di promuovere la salute e il buon funzionamento dell'organismo, prevenire le malattie, fornire assistenza diagnostica o terapeutica e riabilitativa a pazienti, gruppi o comunità nel quadro del rispetto dei valori etici e deontologici. Tutto ciò rientra nei doveri del medico o deve avvenire sotto la sua diretta supervisione e/o prescrizione". Tale definizione sembra però troppo ampia e aspecifica. Le medesime finalità si ritrovano infatti, parlando di assistenza infermieristica, al comma 2 del D.M. n. 739/1994, e nuovamente ribadite nella Legge n. 251/2000.

L'esercizio abusivo di professione è un reato previsto dall'Art. 348 del Codice Penale, che si configura come una cosiddetta norma penale in bianco, presupponendo l'esistenza di altre norme giuridiche che regolino l'esercizio professionale. Nell'attuale contesto normativo italiano non esiste alcuna norma giuridica che definisca in modo preciso il cosiddetto atto medico, di conseguenza il confine tra atto medico e atto infermieristico 
deve essere individuato in relazione alla formazione di base e post-base ricevuta dal singolo professionista (Benci, 2008). Da ciò deriva la necessità di predisporre uno specifico percorso di formazione e di certificazione delle competenze dell'infermiere di "See and Treat".

A quanto detto si può aggiungere che una moderna lettura degli Art. 357 e 358 Codice Penale, in merito alla nozione di pubblico ufficiale, lascia intendere che tutti i professionisti sanitari sono considerabili sia pubblici ufficiali, sia incaricati di pubblico servizio, in quanto ciò che connota l'una o l'altra funzione è la specifica attività realizzata in un dato momento e in un dato contesto (Casati et al., 2009). In merito si è chiaramente pronunciata la Corte di Cassazione, secondo la quale il pubblico ufficiale è colui che "permanentemente o temporaneamente, gratuitamente o con retribuzione, volontariamente o non, esercita una pubblica funzione, intesa questa in senso oggettivo o funzionale e non soggettivo. Ne consegue che tale deve considerarsi il sanitario che presta la sua opera professionale in favore di coloro che possono fruire dell'assistenza pubblica, in quanto svolge un'attività che oltre ad essere connotata intrinsecamente da un evidente interesse pubblico, qual è quello della tutela alla salute, è a lui attribuita, dall'ordinamento dello Stato" (Art. 357 Codice Penale, integrato da Corte di Cassazione, Sentenza N. 8987/1988).

\section{CONCLUSIONI}

Il Pronto Soccorso è oggi sempre più oberato da richieste di prestazioni improprie, senza alcun carattere di urgenza, con un notevole aumento dei tempi di attesa per la visita medica e con il rischio che non vengano tempestivamente erogate prestazioni sanitarie urgenti al paziente critico. L'introduzione di un percorso di "See and Treat" rappresenta una possibile strategia di risposta a queste esigenze.

L'infermiere di "See and Treat" si configura come un infermiere esperto, adeguatamente formato e certificato attraverso una valutazione formale delle specifiche competenze acquisite, che, nella gestione dei problemi clinici minori, opera nel rispetto di protocolli clinico-assistenziali condivisi.

Appaiono quindi prive di ogni fondamento le accuse, mosse dall'Ordine dei Medici di Bologna, di violazione dell'Art. 117, comma 3, Costituzione Italiana, poiché la Regione Toscana non ha indebitamente creato una nuova figura professionale sanitaria, ma ha semplicemente introdotto specifiche prestazioni infermieristiche, afferenti all'area critica, come individuato dal D.M. n. 739/1994, nel pieno rispetto della potestà legislativa concorrente in materia sanitaria tra Stato e Regioni. Inoltre, per quanto riguarda l'accusa di esercizio abusivo della professione medica, neppure in questo caso sembrano ravvisarsi elementi di illegalità, in virtù dell'adozione di protocolli clinico-assistenziali, diagnostici e terapeutici di responsabilità condivisa tra medici ed infermieri. Come affermato da Benci (2008), l'autonomia e le responsabilità di queste due diverse e paritetiche figure professionali si mantengono ben distinte. Da un lato, il medico partecipa alla formazione degli operatori e alla standardizzazione delle procedure terapeutiche, contribuendo così all'erogazione di prestazioni sanitarie appropriate, dall'altro l'infermiere rimane invece responsabile degli atti che pone in essere.

Come affermato dall'Avvocato Barbieri "troppo spesso occupati e preoccupati dalla (inesistente) intrusione dell'infermiere negli spazi medici, si corre il rischio di non censurare le intromissioni del medico in un campo che non è medico ma è, appunto, infermieristico, dove l'infermiere non è l'esecutore di atti decisi da altri, ma è il responsabile, tra le altre e a titolo esemplificativo, dell'identificazione dei bisogni di assistenza infermieristica e della ricerca degli strumenti, dei metodi, delle competenze e delle tecniche tese a fornire una risposta a tali bisogni. Se è indiscutibile che esistono atti medici esclusivi, o forse più correttamente, atti sanitari praticabili in via esclusiva dal medico, deve riconoscersi come esistono atti sanitari praticabili in via esclusiva dagli infermieri, qualificabili atti infermieristici, circa i quali il medico non può intromettersi dal momento che sono di esclusiva competenza infermieristica. [...] Occorre distinguere tra interventi infermieristici autonomi e interventi infermieristici su prescrizione medica, laddove su prescrizione medica non può significare su controllo o supervisione del medico ma piuttosto, in maniera ben più complessa $\mathrm{e}$ articolata, come l'infermiere, in collaborazione col medico, garantisce e quindi assicura la corretta applicazione delle procedure diagnostico-terapeutiche, assumendosi la diretta responsabilità di tale correttezza" (Barbieri, 2010).

\section{BIBLIOGRAFIA}

American Academy of Nurse Practitioner (2010). Frequently Asked Questions. Why choose a nurse practitioner as your healthcare provider? [on-line] da: http://aanp.org/NR/rdonlyres/A1D9B4BD-AC5E45BF-9EB0-DEFCA1 123204/4710/2011FAQsw hatisanNPupdated.pdf, consultato il 16/05/2011

American College of Emergency Physicians (2003). Topic physician assistant-nurse practitioners [on line] da: http://www.acep.org/workarea/downloadasset. aspx?id=9092, consultato il 16/05/2011

Bambi, S., Giusti., M., Becattini, G. (2008). See and Treat in pronto soccorso: dal medico all'infermiere con 
competenze avanzate, una revisione della letteratura. Assistenza Infermieristica e Ricerca, 27(3), 136-144

Barbieri, G., Pennini, A. (2008) Le responsabilità dell'infermiere. Roma: Carocci editore

Barbieri, G. (2010) Le leggi parlano chiaro: le attività sono distinte e autonome. Articolo pubblicato su Il Sole 24 ore Sanità, 3 agosto-6 settembre 2010 pp. 20-21

Benci, L. (1999). Professioni sanitarie... non più ausiliarie. Rivista di diritto delle professioni sanitarie, 1, 3-10

Benci, L. (2008). I principali reati a carico dell'infermiere. In: Aspetti giuridici della professione infermieristica. Milano: McGraw-Hill, 85-90, 146-150

Bullard, M.J., Unger B., Spence J., Grafstein E. (2008) Revisions to the Canadian Emergency Department Triage and Acuity Scale (CTAS) adult guidelines. Canadian Journal of Emergency Medicine, 10(2): 136142

Carter, A.J.E., Chochinov, A.H. (2007). A systrematic review of the impact of nurse practitioners on cost, quality of care, satisfaction and wait times in the emergency department. Can. J. Emerg. Med., 9(4), 286-295

Casati, M., Maricchio, R. (2009). La documentazione infermieristica: riflessioni operative e giuridiche. I Quaderni de L'Infermiere, N. 24 [on line] da: http://213.203.141.50/pubblicazioni/archivioriviste/de ttaglioIndiceRivista.asp?IDRivista $=105$, consultato il 03/03/2012

Department of Health (2011). Statistical press notice: A\&E statistics - quarterly update. [on-line] da: http://www.dh.gov.uk/en/MediaCentre/Pressreleases /DH_126739, consultato il 16/05/2011

Emergency Nurses Association (2008). Competencies for nurse practitioners in emergency care. [on line] da: h t t p : / / www.ena.org/nursing/advanced/ ENANPCompetenciesDec2008.pdf, consultato il $16 / 05 / 2011$

Fordyce J., Blank F.S.J., Pekow P., Smithline H.A., Ritter G., Gehlbach S., Benjamin E., Henneman P.L. (2003). Errors in a busy emergency department. Annals of Emergency Medicine, 42(3), 324-333

Hart L., Mirabella, J. (2009). A patient survey on emergency department: use of nurse practitioner. Advanced Emergency Nursing Journal, 31(3), 228-235

Ieraci S., Digiusto E., Sonntag .P, Dann L., Fox D. (2008). Streaming by case complexity: evaluation of a model for emergency department fast track. Em. Med. Australasia, 20, 241-249

Jennings N., Lee G., Chao K., Keating S. (2009). A survey of patient satisfaction in a metropolitan emergency department: comparing nurse practitioners and emergency physicians. International Journal of Nursing Practice, 15, 213-218

Kwa P., Blake D. (2008). Fast track: has it changed patient care in the emergency department? Em. Med.
Australasia, 20: 10-15

McCarthy M.L., Zeger S.L., Ding R., Levin S.R., Desmond J.S., Lee J., Aronsky D. (2009). Crowding delays Treatment and lengthens emergency department length of stay, even among high-acuity patients. Annals of Emergency Medicine, 54(4): 492-503

Ministero della Salute (2005). Compendio del Servizio Sanitario Nazionale, Ottobre 2005. [on-line] da: http://www.salute.gov.it/servizio/pubblicazioni/ COMPENDIO \%202001_2003.pdf, consultato il $16 / 05 / 2011$

Nash K., Zachariah B., Nitschman J., Psencik B. (2007). Evaluation of a fast track unito $\mathrm{f}$ a university emergency department. J. Emerg. Nursing, 33(1): 14-20

National Health Service (2003). The NHS Modernization Board's Annual Report 2003. [on-line] da: http://www.dh.gov.uk/prod_consum_dh/groups /dh_digitalassets/@dh/@en/documents/digital asset/dh_4064832.pdf, consultato il 16/05/2011

Ordine dei Medici Chirurghi e degli Odontoiatri di Bologna. Esposto a carico delle Regioni Emilia Romagna e Toscana per profili di interesse di possibili violazioni da parte di enti, organi e/o soggetti di riferimento anche organizzativo - degli art. 348 C.P. e art. 8 Legge n. 175/94. (05/10/2010)

Quattrini, V., Swan, B.A. (2011). Evaluating care in emergency department fast track. J. Em. Nursing, 37(1), $40-46$

Regione Toscana. Delibera n. 958 del 17 dicembre 2007. Proposta di sperimentazione del modello "See and Treat" in Pronto Soccorso come modello di risposta assistenziale alle urgenze minori. Approvazione documento.

Regione Toscana. Delibera n. 449 del 31 marzo 2010. Revisione del progetto di sperimentazione del modello "See and Treatt" in Pronto Soccorso.

Rogers, T., Ross N., Spooner D. (2004). Evaluation of a "See and Treat" pilot study introduced to an emergency department. Accident and Emergency Nursing, 12: 24-27

Royal College of Nurses (2007). Advanced Nurse Practitioner competencies. [on-line] da: http://www.rcn.org.uk/ _data/assets/pdf_file/0014/114503/ RCN_ANP_competencies_for_July_07_AU_ Board.pdf, consultato il 16/05/2011

Silvestro, A. (2010) Il punto di vista dell'IPASVI: A ciascuno il proprio livello di efficienza. Il Sole 24ORE-Sanità, 3 agosto-6 settembre 2010

Unione Europea dei Medici Specialisti (2007). Il giornale della previdenza dei medici e degli odontoiatri. Anno IX, n. 6.

Warren, D.W., Jarvis A., LeBlanc L., Gravel J. (2008) Revisions to the Canadian Triage and Acuity Scale Paediatric Guidelines. Canadian Journal of Emergency Medicine, 10(3), 224-232 